\title{
Thermal ignition in rectangular and triangular regions
}

\author{
M.J. Sexton* C. Macaskill* B.F. Gray*
}

(Received 7 August 2000)

\begin{abstract}
When cellulosic materials such as cotton, hay, sawdust or bagasse (sugar-cane residue) are stored in sufficiently large quantities they may self-heat with the possibility of spontaneous ignition. Mathematically, there is a bifurcation to the burning state if ignition occurs. It is important to know the critical values of the basic physical quantities,
\end{abstract}

*School of Mathematics and Statistics, The University of Sydney, NSW 2006, Australia. mailto: jane@maths.usyd.edu.au, mailto:C.Macaskill@maths .usyd.edu.au, mailto:bgray@laurel.ocs.mq.edu . au

${ }^{0}$ See http: //anziamj. austms.org.au/V42/CTAC99/Sext for this article and ancillary services, (c) Austral. Mathematical Soc. 2000. Published 27 Nov 2000. 
such as the ambient temperature or characteristic size of the selfheating sample, at which the bifurcation to the burning state takes place. The solution method for this class of problem depends strongly on the domain under consideration.

Here we consider triangular and rectangular domains with the appropriate mixed boundary conditions. The governing PDEs for the time-dependent problem can be solved by the method of lines, with finite difference schemes used for the discretisation of the spatial derivatives. Any suitable ODE solver can be used for the time integration, so that stiff problems such as those that arise naturally in combustion problems are easily dealt with. In addition, with this approach the steady-state equations are readily extracted and hence the bifurcation structure describing the criticality of the material can be calculated without difficulty. We demonstrate the crucial role played by the boundary conditions in determining, for example, the location of the point of maximum heating.

\section{Contents}

1 Introduction

C1285

2 Mathematical model

C1287

3 Numerical solution methods

C1289

4 Results

C1291 


\section{Introduction}

Self-heating is a process where a material increases in temperature due to the release of heat from ongoing chemical reactions and without drawing heat from its surroundings. The conditions which determine whether self-heating may lead to spontaneous ignition include the rate of internal heat generation and the body's insulation properties, i.e. rate of air supply and heat release. Spontaneous ignition is a major concern because of the dangers associated with fires and the associated destruction or spoilage of the stored product.

While ignition characteristics or criticality for a given stored material depend on a combination of properties including ambient temperature, surface characteristics and the chemistry of the material itself, the geometry is particularly relevant with regard to questions of physical storage. When all other properties are unchanged a small body will lose heat far more quickly than a large body of the same material, with the surface/volume ratio playing a crucial role in defining criticality. 
In this paper, we will be concerned with the thermal ignition problem (including the effects of oxygen consumption) for triangular and rectangular regions and the determination of the critical size of the sample as a function of the base boundary condition. That is, we will look at the range of possibilities for the base boundary condition from fully insulated (Neumann condition) through to the case where the base is set to a given temperature (Dirichlet condition).

Gray, Merkin and Wake [10] have studied the steady-state equations for this model but defined in the slab and showed how the steady-state temperature varied with the ambient temperature. They also showed that as the parameter related to the rate of heating was changed, the steady-state curve crossed the $u_{a}=0$ axis leading to disjoint bifurcation curves thereby giving the non-physical solution of negative temperature. Where Gray, Merkin and Wake [10] used only Dirichlet boundary conditions, Sadiq and Merkin [13] calculated the same curves using both Dirichlet and Robin boundary conditions. There has been much work on the thermal ignition problem, (see the review of Boddington, Gray and Harvey [5]) particularly defined in one dimensional geometry (Gray and Wake [9], for example) and more recently there has been some computational work for three dimensional geometries (Balakrishnan, Swift and Wake [2] and [3] ).

In studying the ignition problem as defined here, we are particularly interested in steady-state solutions to the model and the determination of critical values for physical quantities such as ambient temperature or characteristic size. However, the time-dependent problem is also of importance as the igni- 
tion process may be understood by analysing the evolution of local behaviour such as temperature 'hot-spots'.

\section{Mathematical model}

The nonlinear PDEs describing the temperature and oxygen concentration in a self-heating material undergoing an oxidation reaction can be described by the following equations, (see Gray, Merkin and Wake [10] for example)

$$
\begin{aligned}
\rho c \frac{\partial T}{\partial t^{\prime}} & =\nabla \cdot(\kappa \nabla T)+Q Z W \exp (-E / R T) \\
\frac{\partial W}{\partial t^{\prime}} & =\nabla \cdot(D \nabla W)-Z W \exp (-E / R T) \text { in } \Omega
\end{aligned}
$$

where $\Omega$ is the domain under consideration. The boundary conditions for this problem may be written as

$$
\kappa \frac{\partial T}{\partial n}+h\left(T-T_{a}\right)=0 \quad \text { and } \quad D \frac{\partial W}{\partial n}+h_{W}\left(W-W_{a}\right)=0 \quad \text { on } \quad \partial \Omega
$$

where $\partial / \partial n$ is the normal derivative on the boundary $\partial \Omega, T_{a}$ is the ambient temperature and $W_{a}$ is the oxygen concentration in the surrounding air. The physical characteristics of the material are the thermal conductivity $\kappa$, density $\rho$, exothermicity $Q$, heat capacity $c$, activation energy $E$ and the heat transfer between the material and its surroundings $h$. The constant $Z$ is the 
pre-exponential factor of the exothermic reaction and $R$ is the universal gas constant. The constants $D$ and $h_{W}$ describe the diffusivity of oxygen in the material and the transfer of oxygen from the material to its surroundings, respectively. The domain $\Omega$ is characterised by a length which we will denote by $l$ (measured in metres). (In one dimension, $l$ could be the length of the interval, for example.) Implementing the following non-dimensionalisation scheme, following Gray and Wake [9] rather than Frank-Kamenetskii [8],

$$
\begin{gathered}
u=\frac{R T}{E}, \quad u_{a}=\frac{R T_{a}}{E}, \quad w=\frac{W}{W_{a}}, \quad t=\frac{t^{\prime} \kappa}{\rho c l^{2}}, \\
\lambda=\frac{Z Q R W_{a} l^{2}}{\kappa E}, \quad \beta=\frac{\kappa E}{D R Q W_{a}}, \quad \gamma=\frac{\kappa}{D \rho c}, \quad B i=\frac{h l}{\kappa}, \quad A=\frac{h_{W} l}{D},
\end{gathered}
$$

the governing equations (1) reduce to

$$
\frac{\partial u}{\partial t}=\nabla^{2} u+\lambda w \exp (-1 / u) \text { and } \gamma \frac{\partial w}{\partial t}=\nabla^{2} w-\beta \lambda w \exp (-1 / u)
$$

and the associated boundary conditions (2) become

$$
\frac{\partial u}{\partial n}+B i\left(u-u_{a}\right)=0 \quad \text { and } \quad \frac{\partial w}{\partial n}+A(w-1)=0 \quad \text { on } \quad \partial \Omega
$$

A body of material releasing heat to its surroundings may achieve a safe steady-state where the temperature of the body reaches some moderate value and stabilises. However, when the rate of heat generation of the material exceeds the rate of heat loss to the surroundings, then ignition can occur. That is, there is a critical value of the ambient temperature or size of the stored material above which ignition will occur. 


\section{Numerical solution methods}

For nonlinear parabolic problems such as these, the method of lines (MOL) is a powerful solution technique, where the spatial derivatives are discretised transforming the governing PDEs into a system of ODEs, (see Hairer, Norsett and Wanner [11] for details.) Usually, the chemistry involved in the combustion process introduces stiffness and/or multiple time scales so the availability of robust stiff ODE integrators gives an advantage to this method. For the thermal ignition problem, the finite element method can also be used with the advantage of the method being the ability to solve the problem in arbitrary geometry, whereas the method of lines is more restricted. However, in preparation for the calculation of solutions of more complex combustion problems where multiple time scales are important, we have adopted the method of lines as our solution technique.

The governing PDEs (3) with boundary conditions (4) are transformed into a system of ODEs using finite differences for the spatial derivatives. It is convenient to make a linear transformation on the spatial variables $(x, y)$ (see Appendix A), such that similar coding techniques can be utilised for the solution of the problems for both the triangular and the rectangular domains.

The spatial temperature nodal points are given by $u_{j, k}, j=1, \ldots, n$ and $k=1, \ldots, m$ so the energy equation in its discretised form can be written as

$$
\frac{d u_{j, k}}{d t}=\frac{C_{1}}{2}\left(\frac{1}{\Delta x^{2}}\left(u_{j-1, k}-2 u_{j, k}+u_{j+1, k}\right)+\frac{1}{\Delta y^{2}}\left(u_{j, k-1}-2 u_{j, k}+u_{j, k+1}\right)\right)
$$




$$
\begin{aligned}
& +\frac{C_{2}}{4 \Delta x \Delta y}\left(u_{j+1, k+1}-u_{j-1, k+1}+u_{j-1, k-1}-u_{j+1, k-1}\right) \\
& +\lambda \exp \left(-1 / u_{j, k}\right)
\end{aligned}
$$

where $C_{1}=1+1 / a^{2}, C_{2}=1-1 / a^{2}, \Delta x=1 /(n-1), \Delta y=1 /(m-1)$ and the equation for the oxygen concentration follows in the same manner. We then solve a system of say $s$ ODEs of the form $(u, w)^{\prime}=z^{\prime}=f(z)$. The number of equations to solve in the rectangular region for example is $s=2 \mathrm{~nm}$. When the base angle of the triangular region is equal to $45^{\circ}$, the coefficients of the cross derivative vanish, ( $\operatorname{since} a=1$ ), so that the form of the diffusive terms for the triangular and square region become identical. The second order approximation for the boundary condition is as follows,

$$
u_{B}=\left(4 u_{B-1}-u_{B-2}+2 \Delta x B i u_{a}\right) /(3+2 \Delta x B i)
$$

where $u_{B}$ is the boundary point and $u_{B-1}, u_{B-2}$ are interior points on the normal to the boundary. In the $\alpha=45^{\circ}$ case, the interior points coincide with the grid points but when $\alpha \neq 45^{\circ}$, an interpolation scheme is then required to find the boundary values.

The steady-state equations are calculated by solving $f(z)=0$ as a function of a bifurcation parameter, $u_{a}$ or $\lambda$ for example. In order to calculate the steady-state curve, we use Newton's method. Solving the steady-state equations in this manner is convenient when the time-dependent solution is also of interest, as the same function $f(z)$ is required. In coding this problem, (we have implemented it in Fortran 90 and MATLAB), all that is required is two subroutine calls: one to the ODE integrator (to solve the time-dependent 
problem) and one to the Newton solver (to solve the steady-state problem). The turning point on the lower stable branch of the steady-state curve determines the critical bifurcation value which is related to ignition (see Gray and Wake [9] for example.) This point corresponds to a saddle-node bifurcation, which could be traced out numerically using Newton's method, for example, although we did not use this approach.

As mentioned previously, the finite element method can also be used as a solution technique. We have used Fastflo [7] to calculate both the timevarying solution and the bottom stable branch of the steady-state solution curve, as did Anderson and Zienkiewicz [1] (using the Frank-Kamenetskii [8] variables and approximating the Arrhenius term for small $u_{a}$ ). This solution method serves as a useful check for the MOL technique.

\section{Results}

Physically, we are interested in determining the bifurcation structure for material stored in a body with triangular and rectangular cross-section with varied base boundary conditions. That is, how does the base boundary condition affect the bifurcations for fixed ambient temperature and what size region is needed so that the material won't ignite? Depending on storage conditions, the material may be thermally insulated at the base, or there may be a purely or partially conducting bottom boundary condition. Therefore, we will vary the Biot number $B i$ from 0 through to $\infty$ to cover these possi- 
bilities. Mathematically, this is equivalent to considering the base boundary condition to vary from the Neumann $\partial u / \partial n=0$, to the Dirichlet case $u=u_{a}$. Here it is assumed that the base is impermeable to oxygen, i.e. $\partial w / \partial n=0$, while on the upper boundaries we put $A \rightarrow \infty$, i.e. Dirichlet conditions. In the Neumann case, a triangular region with base angle $45^{\circ}$ is equivalent to a square region, (using the diagonal of the square as a line of symmetry) which therefore serves as a useful benchmark to test solutions, e.g. compare with the table of critical values from Bowes [6].

Firstly, we will illustrate the steady-state solution curves neglecting oxygen consumption, i.e. $w=$ const $=1$ and then compare these solutions to those when oxygen is consumed. In the following examples, the ambient temperature is fixed at $30^{\circ} \mathrm{C}$ and $\lambda$ is the bifurcation parameter. Physically, we are interested in varying the length $l$ of the region to determine the critical length needed for ignition. As defined in the non-dimensionalisation scheme, $\lambda$ is proportional to $l^{2}$ so in the figures following, we will show the physical quantity $l$ instead of $\lambda$. The Biot number $B i$ is also a function of $l$, and this parameter also needs to be varied with $l$. The characteristic length $l$ is the length of the side of the square so that under the transformation described in Appendix A, $\sqrt{2} l$ is the base length of the triangle, regardless of the base angle. Therefore, with $l$ as the dimensional bifurcation parameter, criticality is described for storage sizes for a fixed ambient temperature. (Equally, we could find the critical ambient temperature for a fixed size.) The remaining constants in the model (3) will be set at $\lambda=7.2 \times 10^{13} l^{2}$ and $\beta=63$, which is characteristic of dry bagasse. 
Figure 1(a) describes the increase in critical length $l$ as the base angle is decreased from $45^{\circ}$ to $30^{\circ}$ with Dirichlet boundary conditions on all edges, i.e. $B i \rightarrow \infty$. (Reading from left to right, the angles are $45^{\circ}, 35^{\circ}$ and $30^{\circ}$.) Physically, as the base angle is decreased, the area of the triangle, (i.e. effectively a measure of the volume in this 2-d model), also decreases thereby allowing heat to be conducted more easily to the boundaries. Figure 1(b) shows the effect on the critical length as a function of the Biot number in the bottom boundary condition (see equation (4)). As the Biot number $B i$ is varied from 0 through to $\infty$, the critical length $l$ of the region increases in value. Physically, in the $B i \rightarrow \infty$ limit, heat can escape from all boundaries, rather than from only the upper boundaries as in the $B i=0$ case. The bottom branches of the steady-state curves shown in figures 1(a) and (b) describe stable solutions and the dashed lines depict unstable solutions. The unstable solution is important in determining the critical initial condition, or watershed condition, which is the temperature above which the burning state will be realized. Here, we are plotting the maximum temperature against the dimensional bifurcation parameter $l$, and therefore, the burning state is guaranteed when $u(t=0)>u_{\max }$ everywhere. Problems of determining critical initial conditions are discussed elsewhere, see [9] for example.

In the $B i=0$ case, we calculated the critical length as $l_{\text {crit }}=15.1 \mathrm{~m}$ using $n=31$. In the infinite square rod case, Bowes [6] finds $l_{\text {crit }}=14.9 \mathrm{~m}$. Bowes has made the Frank-Kamenetskii [8] approximation in the Arrhenius term in calculating the table of critical values and we have found in associated work that this approximation is very accurate in finding the turning point for the steady-state curve (which describes the critical value). However, in 

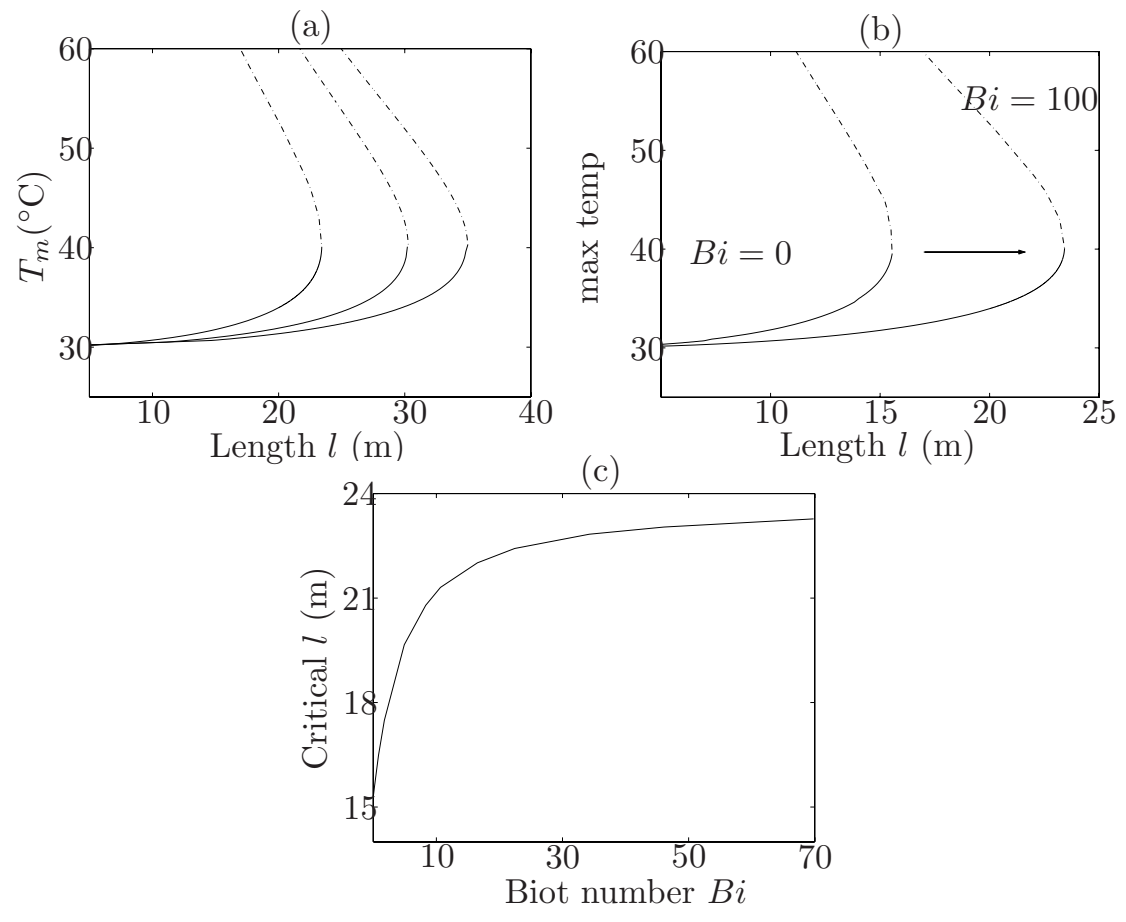

Figure 1: Steady-state temperature solutions for the model (3), (4) with no oxygen consumption where $T_{m}$ is the maximum temperature measured in ${ }^{\circ} \mathrm{C}$. (a) $B i \rightarrow \infty$ with base angles: $45^{\circ}, 35^{\circ}$ and $30^{\circ}$, (from left to right) and (b) $\alpha=45^{\circ}$ with $B i=0$ (i.e. $h=0$, zero flux) and $B i \rightarrow \infty$ (i.e. $h \rightarrow \infty$, perfectly conducting). (c) Critical length for the triangular region with base angle $\alpha=45^{\circ}$ versus $B i$ (by varying the heat transfer coefficient $h$, i.e. $l_{\text {crit }} / \kappa$ fixed). This is a co-dimension 1 curve, i.e. curve of critical conditions. 
determining the watershed condition, it is found that this approximation is inadequate. Even though the prediction of the unstable branch by [8] is quite different to that obtained from the full model, it gives a lower and therefore safer temperature. In addition, Bowes [6] does not include critical values for regions with triangular cross-section, (apart from the one with a base angle of $45^{\circ}$ as it is equivalent to the infinite square rod) and also Dirichlet boundary conditions are assumed for the temperature whereas we can easily alter the boundary conditions to find the critical values. As shown in figures 1(b) and (c), the characteristic scale increases by approximately $57 \%(15.1 \mathrm{~m}$ to $23.7 \mathrm{~m})$ as the Biot number is increased from 0 to the limit $B i \rightarrow \infty$. Figure $1(\mathrm{c})$ shows how the critical scale and Biot number are related. Noting that $B i$ is proportional to $l$, then figure $1(\mathrm{c})$ shows that Dirichlet conditions are approximated when $B i \approx 70$. Using the definition of $B i$, the ratio of heat transfer to thermal conductivity is then $h / \kappa \approx 3$. The relationship between the characteristic length and Biot number in figure 1(c) takes the same form as shown in Balakrishnan, Swift and Wake [3] for the infinite square rod where they plotted the Biot number against $\lambda^{\prime}$ (the value of $\lambda$ which makes the steady-state curve disjoint).

Figures 1(a)-(c) show the steady-state solutions when oxygen consumption is ignored. When the consumption and diffusion of these processes are allowed, it can be seen in Figure 2 that the critical length of the region has only slightly increased. In addition, the high (stable) burning temperature is much less when oxygen is included in the model, (not shown on these diagrams but reduced from a burning temperature greater than $1000^{\circ} \mathrm{C}$ to approximately $300^{\circ} \mathrm{C}$.) This has been shown by Gray, Merkin and Wake [10] 
(a)

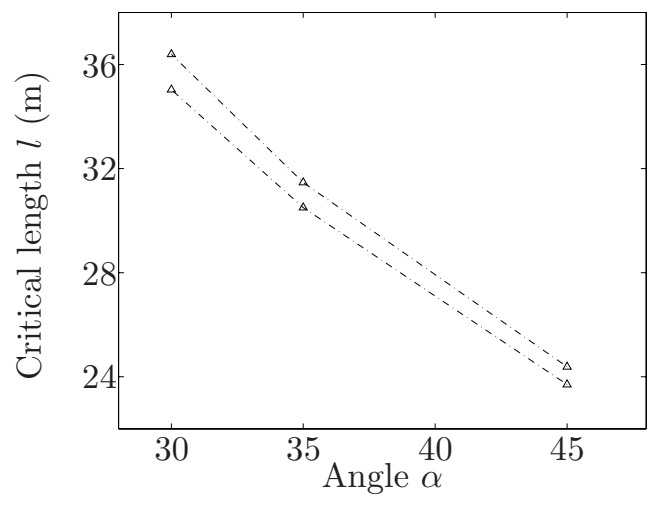

(b)

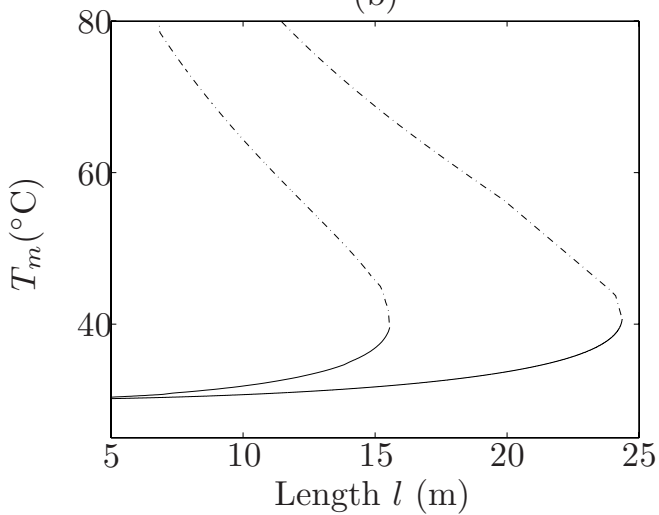

Figure 2: (a) Comparison of critical length $l$ versus base angle of triangle for model with (upper) and without oxygen (lower) consumption. The small triangles depict critical lengths for the base angles of $45^{\circ}, 35^{\circ}$ and $30^{\circ}$. (b) Steady-state temperature solutions with oxygen consumption for $B i=0$ and $B i \rightarrow \infty$ for $\alpha=45^{\circ}$, i.e. $h=0$ and $h \rightarrow \infty$ respectively (reading from left to right). 
for an exothermic reaction in a porous slab. This is to be expected as oxygen will be completely consumed in the burning state, therefore limiting the reaction term. We also note that the temperature at which ignition occurs $\left(40^{\circ} \mathrm{C}\right.$ from Figure $\left.1(\mathrm{a})\right)$ is much lower than found in [10] where ignition temperatures vary from $u=0.05$ to 0.07 (in non-dimensional terms) which corresponds to temperatures very much greater than $100^{\circ} \mathrm{C}$. They used $\lambda$ in the range of $5 \times 10^{4}$ to $10^{6}$, where we have used a much higher value of $\lambda=7.2 \times 10^{13} l^{2}$ (relevant in particular for our ongoing interest of bagasse piles [12].) This critical temperature is typical for hay, woodchips and other agricultural residues stored in these sizes which have ignition temperatures of $30-40^{\circ} \mathrm{C}$.

As mentioned earlier, it is often instructive to solve the time-dependent problem to study the process of ignition. Figures 3 and 4 illustrate the spatial temperature evolution for a non-burning and burning case respectively when the base is insulated for both temperature and oxygen and with ambient conditions on the upper sides. In order for the material not to burn, we must choose a value of $l$ corresponding to the base stable part of the steady-state curve shown in Figure 2(b).

As our example, we take the base angle of the triangular region to be $\alpha=45^{\circ}$ which corresponds to the left-hand steady-state curve in Figure 2(b). Here, we choose $l=15 \mathrm{~m}$ to illustrate the non-burning case and $l=25 \mathrm{~m}$ for the burning case. The initial conditions for each case are set to the ambient values for temperature and oxygen: $u(t=0)=u_{a}$ and $w(t=$ $0)=1$, and the boundary conditions are set to Neumann for the base and 

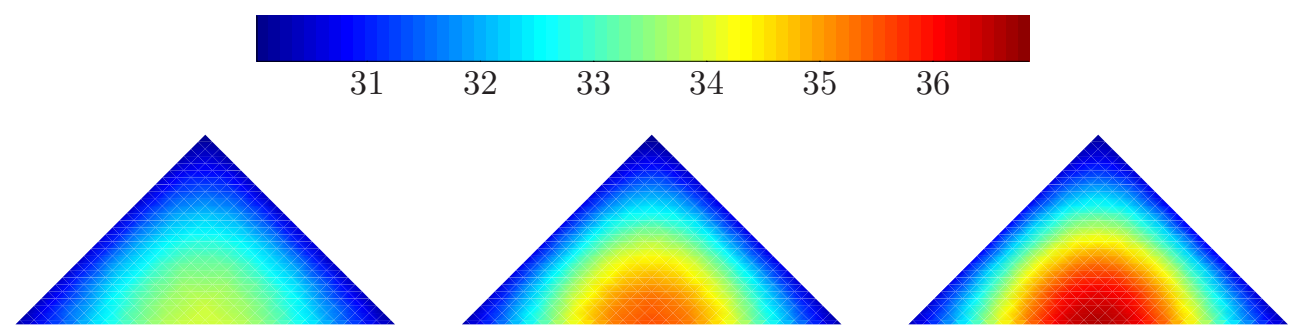

Figure 3: Spatial temperature evolution for the non-burning scenario. The times are $t=87.7,173.9$ and 365 days, and advance from left to right.

Dirichlet on the sides for both temperature and oxygen. In the non-burning case, the maximum temperature is seen to occur at the centre of the base boundary. In this case, the triangular and square region are equivalent (as discussed earlier), and it is known that the maximum temperature occurs in the centre of a square with Dirichlet boundary conditions. The spatial solutions evolve to a stable non-burning state as seen in Figure 3 with a maximum temperature of $36.85^{\circ} \mathrm{C}$.

The burning scenario is illustrated in Figure 4. Here we see the temperature initially increases in the same way as for the non-burning case (that is, heat is generated near the centre of the base then conducted towards the edges of the triangular region) and as the heat is lost to the environment is exceeded by the heat produced by the reaction, the temperature within the region continues to increase. At this point, the oxygen levels within the region have dropped to almost zero, yet oxygen is continuously provided 

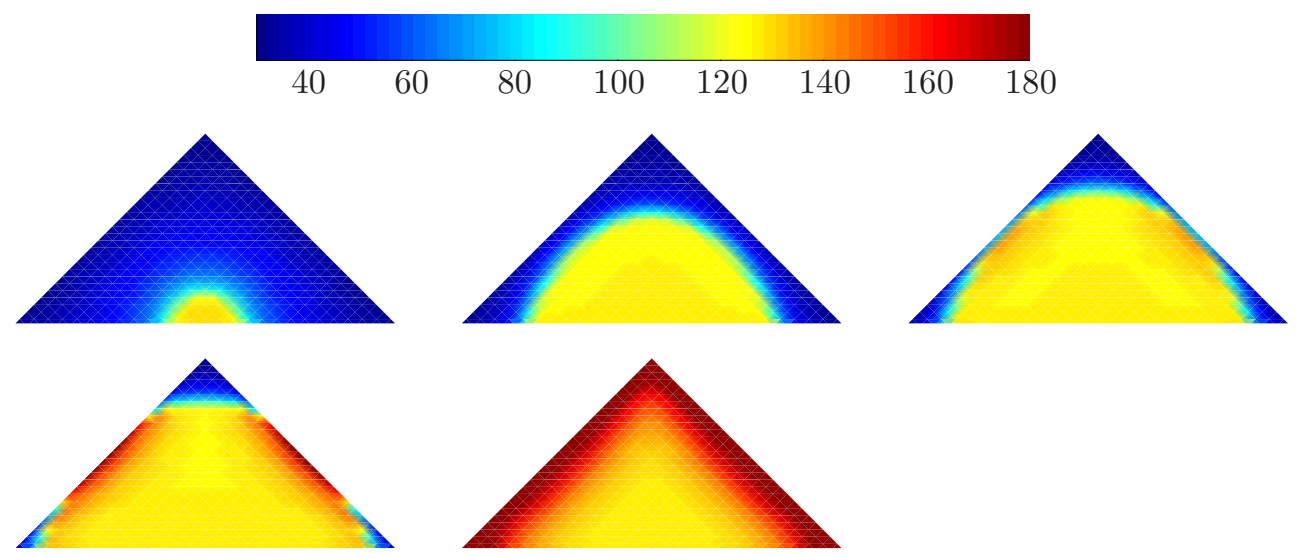

Figure 4: Spatial temperature evolution for the burning scenario. The times are $t=183.2,200.1,207,211$ and 225 days, advancing from left to right and top to bottom.

through the upper boundaries allowing continued burning near these edges. The third frame of Figure 4 corresponds to the time at which the maximum temperature (at this stage, approximately $130^{\circ} \mathrm{C}$ ) is found near each of the centres of the upper edges. For each of the upper edges this maximum then broadens out along the edge in both directions until in the last frame, when the temperature is roughly constant on the upper edges, there is a further jump to the final maximum burning temperature of about $240^{\circ} \mathrm{C}$. Figure 5 shows the maximum temperature evolving in time for the (a) non-burning and (b) burning cases respectively. The exponential time evolution for the 
(a)

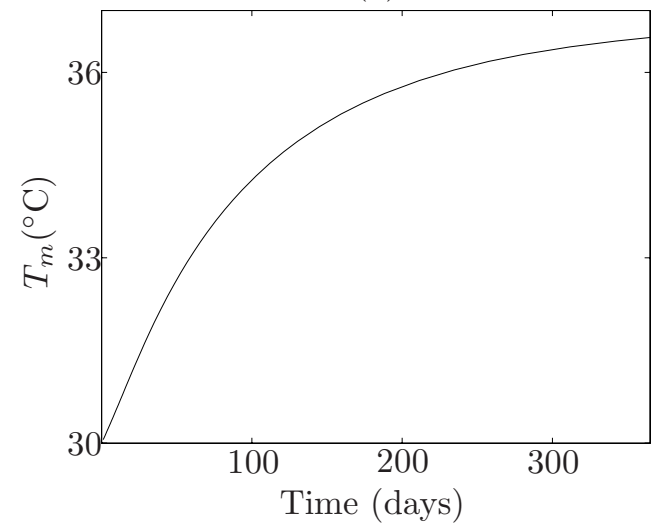

(b)

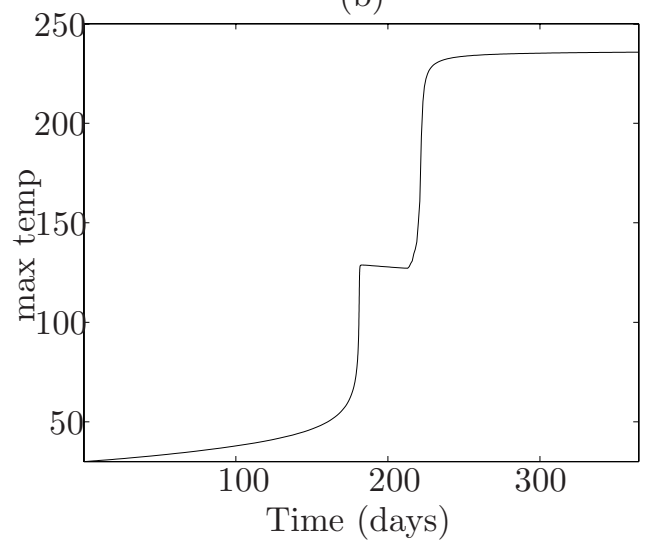

Figure 5: Evolution of the maximum temperature $T_{m}$ (measured in ${ }^{\circ} \mathrm{C}$ ) of the reaction for the (a) non-burning and (b) burning state. 
non-burning state shows the temperature approaching the stable steady-state from below. In the ignited case shown in Figure 5(b), the initial period shows a steady increase in temperature before reaching approximately $130^{\circ} \mathrm{C}$ and then accelerating to the ignited state. The plateau in Figure 5(b) at around $130^{\circ} \mathrm{C}$ followed by the sudden rise to the final ignited steady-state corresponds to the time range of the frames shown in Figure 4. Here, the heat generation of the reaction exceeds the heat loss to the environment, and oxygen is almost completely consumed in the interior. However, burning continues near the edges as oxygen is continuously supplied.

\section{Discussion}

To accurately model most combustion problems, many variables are needed to fully describe the system's behaviour which includes complicated reaction kinetics and multiple time-scales. Due to the availability of robust stiff ODE integrators, the MOL technique is well suited to combustion problems. The range of domains for which this strategy can be implemented however is rather limited, but it has recently been shown that the MOL technique can be introduced in the finite element framework as described by Berzins and Ware [4]. 


\section{References}

[1] C.A. Anderson and O.C. Zienkiewicz. Spontaneous ignition: finite element solutions for steady and transient conditions. J. Heat Transfer, 398-404, 1974. C1291

[2] E. Balakrishnan, A. Swift, and G.C. Wake. Multiple solutions in hollow geometries in the theory of thermal ignition. Appl. Math. Lett., 10:41-46, 1997. C1286

[3] E. Balakrishnan, A. Swift, and G.C. Wake. Critical values for some non-class A geometries in thermal ignition theory. Mathl. Comput. Modelling, 24:1-10, 1996. C1286, C1295

[4] M. Berzins and J.M. Ware. Solving convection and convection-reaction problems using the method of lines. Appl. Numerical Mathematics, 20:83-99, 1996. C1301

[5] T. Boddington, P. Gray, and D.I. Harvey. Thermal theory of spontaneous ignition: criticality in bodies of arbitrary shape. Phil. Trans. Roy. Soc. of London, 270:467-506, 1971. C1286

[6] P.C. Bowes Self Heating: Evaluating and Controlling the Hazards. H.M. Stationery Office, 1984. C1292, C1293, C1295

[7] CSIRO Fastflo: Flexible finite element software for research innovation and design. [Computer program] Version 2.1, 1998. C1291 
[8] D.A. Frank-Kamenetskii. Diffusion and Heat-Transfer in Chemical Kinetics. Princeton University Press, NJ, 1955. C1288, C1291, C1293, C1295

[9] B.F. Gray and G.C. Wake. Critical initial conditions for thermal ignition. Mathl. \& Comput. Modelling, 18:65-75, 1993. C1286, C1288, C1291, C1293

[10] B.F. Gray, J.H. Merkin, and G.C. Wake. Disjoint bifurcation diagrams in combustion systems. Mathl. Comput. Modelling, 15:25-33, 1991. C1286, C1286, C1287, C1295, C1297

[11] E. Hairer, S.P. Norsett, and G. Wanner. Solving Ordinary Differential Equations, vol 1 Nonstiff Problems. Springer Series in Computational Mathematics 8. Springer-Verlag, Berlin, 1987. C1289

[12] C. Macaskill, M.J. Sexton, and B.F. Gray. A reaction-diffusion model of stored bagasse. J. Austral. Math. Soc. Ser. B. To appear. C1297

[13] M.A. Sadiq and J.H. Merkin. Combustion in a porous material with reactant consumption: the role of the ambient temperature. Mathl. Comput. Modelling, 20:27-46, 1994. C1286 


\section{A Appendix}

Let the square coordinates $(x, y)$ be defined on $x \in[0,1]$ and $y \in[0,1]$ and we wish to map the $(x, y)$ coordinates to the triangular coordinates $(p, q)$. Define the mapping by

$$
p=\frac{1}{\sqrt{2}}(x+y) \quad q=\frac{a}{\sqrt{2}}(y-x)
$$

where $a=\tan (\alpha)$ and $\alpha$ is the base angle of the triangle. In $(x, y)$ coordinates, the diffusive term is $\nabla^{2} u=u_{x x}+u_{y y}$, so in the triangular domain,

$$
\nabla^{2} u=u_{p p}+u_{q q}=\frac{1}{2}\left(\left(u_{x x}+u_{y y}\right)\left(1+\frac{1}{a^{2}}\right)+2 u_{x y}\left(1-\frac{1}{a^{2}}\right)\right) .
$$

